\begin{tabular}{|c|l|}
\hline Title & Nach Chomsky: Überlegungen zu einer symboltheoretisch fundierten Linguistik \\
\hline Sub Title & \\
\hline Author & Stetter, Christian \\
\hline Publisher & 慶應義塾大学日吉紀要刊行委員会 \\
\hline Publication year & 2003 \\
\hline Jtitle & 慶應義塾大学日吉紀要. ドイツ語学・文学 No.35 (2003. 2),p.141-172 \\
\hline JaLC DOI & \\
\hline Abstract & \\
\hline Notes & \\
\hline Genre & Departmental Bulletin Paper \\
\hline URL & https://koara.lib.keio.ac.jp/xoonips/modules/xoonips/detail.php?koara_id=AN100 \\
32372-20030210-0141
\end{tabular}

慶應義塾大学学術情報リポジトリ(KOARA)に掲載されているコンテンツの著作権は、それぞれの著作者、学会または出版社/発行者に帰属し、その 権利は著作権法によって保護されています。引用にあたっては、著作権法を遵守してご利用ください。

The copyrights of content available on the KeiO Associated Repository of Academic resources (KOARA) belong to the respective authors, academic societies, or publishers/issuers, and these rights are protected by the Japanese Copyright Act. When quoting the content, please follow the Japanese copyright act. 


\section{Nach Chomsky}

\section{- Überlegungen zu einer symboltheoretisch fundierten Linguistik -- -}

\section{Christian STETTER}

Inhalt:

0 . Am Ende des Chomsky-Paradigmas

1. Stationen einer Revision der Sprachtheorie

2. Zwischenbilanz

3. Konsequenzen

4. Essentials einer nach-chomskyschen Sprachtheorie

\section{Am Ende des Chomsky-Paradigmas}

Die theoretische Gestalt, die die Sprachwissenschaft im 20. Jahrhundert gewonnen hat, ist Resultat einer Entwicklung, die man durchaus zurecht unter den Titel einer Entrhetorisierung der Geisteswissenschaften subsumieren kann. Schon bei F. de Saussure und im frühen Strukturalismus, dezidiert dann bei Noam Chomsky ist eine Vernachlässigung der Performanz als theoretischen Gegenstandes der Disziplin zu konstatieren. ${ }^{12}$ Wie immer dies begriindet sein mochte - diese Entwicklung hat die Linguistik nicht nur von der Philologie emanzipiert, sondern sie jedem rhetorischen Verständnis von Sprache entfremdet. Denn die Performanz steht im Zentrum einer jeden Rhetorik. Darstellung, so die Quintessenz der Symboltheorie Nelson Goodmans, ist an Performanz gebunden. ${ }^{2)}$ Von dieser Ebene - in Saussures

1) Zwar spricht Saussure auch von einer linguistique de la parole, doch diese Überlegungen gewinnen kaum an Kontur gegenüber denen, die auf den systematischen Charakter der langue zielen. 
Terminologie der der parole - hat sich die Linguistik mit der von Chomsky eingeleiteten Wende der Disziplin hin zu einer Kognitions-wissenschaft so weit entfernt, daß die sprachliche Performanz aus dem Kreis möglicher Gegenstände der Sprachtheorie gänzlich ausgeschlossen scheint.

Wäre dieser Weg unstrittig und erfolgversprechend, so bestünde kaum Anlaß, über eine Revision des theoretischen Ansatzes nachzudenken. Aber das chomskysche Paradigma hat sich spätestens mit dem Minimalistischen Programm so unübersehbar in Aporien verstrickt, die unlösbar scheinen, daß in der Tat Anlaß besteht, über Alternativen nachzudenken. ${ }^{3)}$ Eine dieser Aporien sei hier genauer betrachtet. Sie ist, im Zentrum der chomskyschen Theorie angesiedelt, logischer Natur: die Käfer-in-der-Schachtel-Aporie: ${ }^{4}$ )

Auch und gerade als eine Theorie über den sogenannten universalgrammatischen Kern der dem Menschen angeborenen Sprachkompetenz bleibt die Theorie der Universalgrammatik (UG) eine empirische Theorie. Ihre Gültigkeit hat sich - dem eigenen Anspruch zufolge - experimentell zu bewähren. Das empirische Design der generativen Theorie baut wesentlich auf der Arbeit mit Beispielsätzen auf. Das braucht hier nicht in extenso ausgeführt zu werden. ${ }^{57}$ Bestätigt werden kann die Annahme von UG-Kategorien, mithin die Plausibilität eine entsprechenden Theorie über diese, nur im Rahmen der künstlichen Erzeugung von Beispielsätzen, deren Wohlgeformtheit oder Agrammatikalität in der jeweiligen „Versuchsanordnung “ auf diese oder jene UG - Kategorie, dieses oder jenes Prinzip der UG zurückgeführt wird. Das Performanz-Resultat wird durch eine hypo-

2) Vgl. Goodman 1997, S. 140: “Prinzipiell ist Erfuillung [eines gegebenen Symbolschemas, Ch. St.] mit einer Inskription verbunden“, d. h. mit der performativen Instantiierung eines Charakters. Goodmans Symboltheorie setze ich im folgenden voraus, ohne sie hier explizit einzuführen. Als knappe Orientierung sei verwiesen auf Goodmans Beitrag „Re-repräsentierte Repräsentation“ in Goodman und Elgin 1989. Zu den Begriffen Symbolschema und Symbolsystem vgl. Goodman 1997, S. 128 ff., Goodman und Elgin 1989, S. $165 \mathrm{ff}$.

3) Vgl. hierzu Stetter 2002.

4) Vgl. hierzu Stetter 1999.

5) Vgl. hierzu grundlegend schon Simon 1974, darauf aufbauend Stetter 1997, Kap. 5. 
thetisch angesetzte Kompetenz - Struktur , erklärt' . ${ }^{6}$

Soweit das bekannte Design der generativen Theorie. Gemäß dem von Chomsky bereits in den frühen Aspekten der Syntax-Theorie praktizierten doppeldeutigen Gebrauch des Begriffs "Grammatik“ - das mentale Organ und dessen schriftliches Modell - werden, und dies ist die zweite Besonderheit, die Chomsky in die linguistische Theorie eingeführt hat, syntaktische Kategorien im Rahmen einer Phrasenstrukturgrammatik extensional definiert. ${ }^{7}$ Dies geschieht mithilfe von Phrasenstrukturregeln, die die Form von Ersetzungsanweisungen haben: $A \rightarrow B+C$ : Ersetze das Symbol A durch die Folge „B $+C$ “ bzw. in nichttechnischer Lesart: $A$ ist $B+C$.

Das Anfangssymbol einer solchen Grammatik ${ }^{8)}$ wird in $n$ Expansionen bis zu einer , terminalen' Kette von Symbolen $U+V+\ldots+W$ entwickelt. Diese werden ihrerseits durch sogenannte Lexikonregeln wiederum extensional interpretiert: $\mathrm{U}\{$ der, die das, ... $\}, \mathrm{V}\left\{\right.$ geb $_{-}$, lauf, schlaf,$\left.\ldots\right\}$ usw. ${ }^{9)} \mathrm{U}$ wäre hier eine Menge von Ausdrücken wie der, die das und ähnlichen, die ein grammatisch gebildeter Muttersprachler des Deutschen in geeigneten Kontexten als

6) Diesen Anspruch der generativen Theorie hat sehr klar G. Grewendorf in einer Arbeit verdeutlicht, die zu der hier diskutierten Käfer-in-derSchachtel-Aporie in engem thematischem Bezug steht. Vgl. Grewendorf 1995, S. 60 ff., dazu meine Diskussion der Interpretation, die Grewendorf dort dem Privatsprachenproblem gibt, in Stetter 1999.

7) Das Problem der Transformationsregeln vernachlässige ich hier. Es ist für das Argument belanglos.

8) Hier sind insbesondere zwei Metaregeln zu beachten, die die Logik einer Phrasenstrukturgrammatik regeln:

1. Links vom Ersetzungspfeil “ $\rightarrow$ " darf ein Symbol ( “A”, “B”, “C”, “...") erst erscheinen, nachdem es zuvor rechts vom Ersetzungspfeil eingeführt wurde.

2. Links vom Ersetzungspfeil steht genau ein Symbol.

9) Lexikonregeln sind im strikten logischen Sinne keine Regeln. Der Ausdruck „V $\left\{g e b_{-}\right.$, lauf, schlaf,$\left.\ldots\right\}$ “ besagt - im Unterschied zu Ersetzungsregeln - nicht, durch welches der in der Klammer aufgeführten Ausdrücke das Symbol V zu ersetzen wäre. Er ist somit zu lesen als: geb_ ist V oder lauf_ist V oder schlaf_ ist V ..., und dies ist eine Alternation kategorischer Prädikationen, kein generalisiertes Konditional. 
Artikel oder Relativpronomen erkennen kann.

Ob eine in dieser Grammatik beschriebene Struktur

(1) $\mathrm{A}$

$$
\begin{gathered}
\text { (2) } \mathrm{B}+\mathrm{C} \\
\text { (3) }(\mathrm{E}+\mathrm{F})+(\mathrm{G}+\mathrm{H}) \\
(\mathrm{E}+\mathrm{F})+\mathrm{G}+(\mathrm{I}+\mathrm{K}) \\
\ldots \\
\text { (n) } \mathrm{U}+\ldots+\mathrm{V}+\mathrm{W}+\ldots+\mathrm{Y}+\mathrm{Z}^{10)}
\end{gathered}
$$

'deskriptiv adäquat' ist, entscheidet sich somit erst durch die Interpretation der terminalen Kette durch Lexikonregeln. Diese Interpretation - z. B. der Hans liebt die Inge - ist aber qua geordnete Folge von Wörtern nun kein Element eines wie gearteten Systems mehr, sondern Element der parole, mithin eine Performanzerscheinung, hier ein bestimmter Satz der deutschen Schriftsprache. Dies folgt aus dem logischen Status der Lexikonregel. Die geordnete Folge von Wörtern kann kein Systemelement sein, da aus der Lexikonregel ja nicht hervorgeht, durch welches Element der betreffenden Lexemmenge das Kategoriensymbol zu ersetzen ist. ${ }^{11)}$ Der ganze Ausdruck ist also nicht nach Regeln gebildet, bestenfalls gemäß bestimmten Regeln - ein wesentlicher Unterschied. ${ }^{12}$

Der Übergang von der terminalen Kette der Phrasenstrukturregel zu der folgenden Verkettung von Lexikoneinheiten markiert also in jeder grammatischen Darstellung den Übergang von der Beschreibungsebene der Kompetenz bzw. des Systems zu der der Performanz bzw. der parole. Der jeweils gemäß einer wie auch immer gearteten Regelfolge (1) bis ( $n$ ) erzeugte Ausdruck kann selbst nicht mehr als "Satz im Kopf“ und damit als Element des Systems - oder der Kompetenz - interpretiert werden, weil der Zweck der Darstellung ja darin besteht, anhand des so erzeugten Ausdrucks die Wohlgeformtheit des Ausdrucks beurteilen zu können. Er ist Element einer Menge von Ausdrücken, die extensional betrachtet das bilden, was man eine, öffentliche' Sprache nennt - ergon im Sinne W. von Humboldts.

10) Die Klammern verwende ich hier informell zur Andeutung der "Derivationsgeschichte“.

11) Zurecht hat daher Saussure den Satz als Element der parole begriffen. Vgl. CLG S. 172.

12) Vgl. hierzu Stetter 1997, S. 79 f. 
Beispielsätze gelten der linguistischen Dogmatik als Exempel "gelungener"Anwendung von Regeln. Tatsächlich aber exemplifiziert - in Termen der Symboltheorie Goodmans - der Beispielsatz eine gelungene oder mißlungene Performanz. ${ }^{13)}$ Aus dieser Konstellation resultiert die Käfer-in -der-Schachtel-Aporie: Der generativen Theorie zufolge besteht jede Grammatik aus einem Kern, der Elemente der UG umfaßt, und einer ,Peripherie', deren Elemente einzelsprachliche Besonderheiten repräsentieren. Der Theorie nach sind die Symbole, von denen in einer Phrasenstrukturgrammatik Gebrauch gemacht wird, daher zugleich Namen von Systemkategorien. Ihnen wird durch diese Interpretation, die aus Chomskys doppeldeutigem Gebrauch des Wortes "Grammatik“ resultiert, eine zweite, gänzlich andersartige Extension zugeordnet. Sie referieren nun nicht mehr qua Ersetzungsregel auf die ihnen in dieser zugewiesene Expansion, ${ }^{14)}$ sondern auf mentale Zustände wie "Satz“, „Subjekt-von“, “VP-von“ usw. Mindestens die erste Stufe der Expansion eines Anfangssymbols muß daher ein Element der UG sein. Nun ist aber die Beantwortung der Frage, ob das Symbol zutreffend angesetzt ist, allein abhängig davon, wie es - wie gezeigt im weiteren expandiert und in einen von einem Muttersprachler interpretierbaren Performanzausdruck überführt wird.

Daher kürzt sich die zweite, mentale Extension der Kategoriensymbole im Verfahren der Erzeugung von Beispielsätzen weg wie Wittgensteins Käfer in der Schachtel. ${ }^{15)}$ Was bleibt, ist eine mißverstandene Praxis. Beispielsätze können eine Kompetenztheorie weder verifizieren noch falsifizieren. Beides ist - aus unterschiedlichen Gründen - logisch unmöglich. Tatsächlich gibt der Grammatiker mit dem Erfinden von ,wohlgeformten' und , abweichenden' Paradigmen Proben seines Talents, sinnvolle sprachliche Ausdrücke zu bilden, die in bestimmten Hinsichten geeignet sind,

13) Vgl. hierzu Stetter 1997, Kap. 5, wo ich das Problem linguistischer Beispielsätze allerdings noch nicht symboltheoretisch behandelt habe.

14) In einer Ersetzungsregel $\mathrm{X} \rightarrow \mathrm{Y}+\mathrm{Z}$ erfüllt das Zeichen $" \rightarrow$ “ ja nicht nur die Funktion, dem Symbol „X“ den Ausdruck „Y+Z“ als seine Expansion zuzuordnen. Gleichzeitig weist es $\mathrm{Y}+\mathrm{Z}$ als Extension von $\mathrm{X}$ aus, $\mathrm{X}$ damit als Namen für $Y+Z$. Symboltheoretisch betrachtet ist $Y+Z$ somit eine Exemplifikation dessen, was $\mathrm{X}$ darstellt.

15) Dieses Argument habe ich in Stetter 1999 genauer ausgeführt. 
etwas zu exemplifizieren. Und wenn er gegen das, wohlgeformte'

Peter schenkt dem Bruder zu Weihnachten ein Buch die, abweichenden'

${ }^{*}$ Peter schenkt zu Weihnachten ein Buch dem Bruder

${ }^{*}$ Dem Bruder schenkt ein Buch zu Weihnachten Peter

${ }^{*}$ Es schenkt Peter dem Bruder zu Weihnachten

setzt und das Abweichen mit einem Asterisken markiert, so kann dieses zusätzliche Zeichen doch nicht verhindern, daß diese Sätze als Beispiele gerade nicht miß- sondern gelungen sind und somit offenbar tatsächlich gan $z$ andere Eigenschaften einer wie auch immer gearteten Kompeten $z$ exemplifizieren als dieser in der Theorie zugeschrieben werden, nämlich die zur analogischen Bildung neuer Formen in neuen Kontexten. ${ }^{16)}$

Dies mag hier als Einführung in die Problemlage genügen. ${ }^{17}$ Im Akt der Abspaltung einer idealisierten Kompeten $z$ von einer vorgeblich defektiven Performanz bleibt ironischerweise - der technischen Modellierung des Regelkonzepts geschuldet - die Kompetenz auf der Strecke. Eine Revision des Paradigmas ist - daran kann wohl kaum ein Zweifel sein - unvermeidlich.

\section{Stationen einer Revision der Sprachtheorie}

Eine solche Revision tut gut daran, die wichtigsten Stationen der Entwicklung zu betrachten, die die Linguistik an diesen Punkt gebracht hat. In ihr hat sich ein bestimmtes Bildes der Sprache ausgeprägt. An ihrem Beginn steht die Digitalisierung der Sprache, die mit der Evolution des Alphabets geleistet worden ist. Dies hat den späteren Begriff von Sprache geprägt. Gängigen Mythen über das Alphabet zum Trotz funktioniert dieses

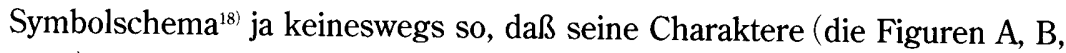
C, ...) als Namen für entsprechende Elemente der oralen Sprache dienten.

16) Vgl. zum Begriff der Exemplifikation Goodman 1997, Kap. 2 und passim.

17) $\mathrm{Zu}$ einer ausführlicheren Darstellung der Aporien dieses Paradigmas vgl. Stetter 1997, Kap. 6 und Stetter 2002a.

18) Den Begriff Symbolschema verwende ich in dem von Goodman präzisierten Sinn. Vgl. Goodman 1997, Kap. 4, und Goodman und Elgin 1989, S. $162 \mathrm{ff}$. 
Denn deren elementare Charaktere, die Wörter, ${ }^{19)}$ sind kontinuierlich modulierte "Laute“, die ihrerseits kontinuierlich miteinander verbunden werden. Vor jeder theoretischen Reflexion über die Schrift hat die besondere Form des Alphabets die spätere Sprachtheorie, die sich in ihrem Bereich entwickelt hat, mit der Hypothek der Zweideutigkeit von Digitalität versus Analogie belastet. ${ }^{20}$

Die klassische Phonologie konstruiert das Phonem als die kleinste distinktive Einheit der oralen Sprache. Doch lohnt es sich, die klassische Doktrin genau zu lesen. Das Phonemsystem einer jeden Sprache baut sich nach dem Prinzip des maximalen Kontrasts auf. ${ }^{21)}$ Der maximale Kontrast , vokalisch' : , konsonantisch' wird realisiert in Artikulationen, die traditionell durch die Merkmale [+ Öffnung] und [+ Sonorität] beziehungsweise [+ Hemmung] und [- Sonorität] beschrieben werden. Doch die gewohnte Lesart, die diese Ausdrücke als Merkmale liest, als Eigenschaften von Einheiten, verdankt sich auch hier der Unterscheidung von System und Performanz. Letztere ist Bewegung, und somit beschreibt das Merkmalbündel [+ Öffnung, + Sonorität] die Erzeugung von Öffnung und von Sonorität und das Merkmalbündel [+ Hemmung, - Sonorität] die Erzeugung von Hemmung bei fehlender Sonorität. Das Phonem ist, so betrachtet, das Schema einer Artikulationsveränderung, in der die Extreme kontinuierlich ineinander übergehen. ${ }^{22}$

Diesen kontinuierlichen Artikulationsfluß bildet der Gebrauch des Alphabets auf Folgen diskreter Elemente ab. Selbst wo diese in Kursivschriften miteinander verbunden werden, repräsentieren die einzelnen Inskriptionen Folgen effektiv differenzierbarer Elemente. ${ }^{23}$ In der Praxis dieser Abbildung wird der Begriff der phonē grammatisch gefaßt und diese damit der Sache nach als digitales Schema konzipiert, auch wenn Theorie wie Praxis der Grammatik diesen Begriff bis in die Neuzeit hinein nicht kennen. Und an dieser Stelle ist festzuhalten, daß sich weder die strukturale

19) Die Morphologie vernachlässige ich hier der Einfachheit halber.

20) Den klassische Beleg liefert Platons Theaitetos mit dem sokratischen Mythos von den einfachen, nichts mehr bedeutenden Urelementen. Vgl. Theaitetos $201 \mathrm{e} f \mathrm{fr}$.

21) Vgl. zum folgenden Jakobson 1969 und Eisenberg 1998, Kap. 2 und 3.

22) Vgl. hierzu Stetter 2002. 
noch gar die generative Linguistik des 20. Jahrhunderts jemals Rechenschaft über diese schriftinduzierte Präformation ihres Sprachbildes abgelegt hat. ${ }^{24}$

Die technē grammatikē liefert Platon das Modell für die Konzeption einer formal wie diese verfahrenden technē dialektikē, welche - das ist für unsere Rekonstruktion von Bedeutung - der Rhetorik entgegengesetzt wird. ${ }^{25}$ ) Überzeugen kann ein Argument in philosophischer Hinsicht nur dann, wenn es widerspruchsfrei verteidigt werden kann. Alle anderen Überzeugungsmittel, zumal solche, die nicht an die Vernunft appellieren, sondern an Sinnlichkeit oder Gefühl des angesprochenen Publikums, werden aus dem Sprachspiel der Philosophie verbannt. Die Problematik dieser Diskreditierung der Rhetorik braucht hier nicht weiter erörtert zu werden. Das Faktum steht ebenso außer Zweifel wie die philosophische Rehabilitation, die die Rhetorik bei Aristoteles erfährt. Dies allerdings nun auf der Basis eines logischen Verständnisses philosophischer Dialektik. ${ }^{26)}$

Formal verfährt die neue Kunst, die Logik, insofern, als sie der Repräsentation, die die Sprache in Gestalt der Schrift erfährt, den Gedanken der Aufspaltung des vorher untrennbaren Zusammenhangs von Gedanke und logos in Symbole entnimmt, die etwas bedeuten allein insofern, als sie Namen für anderes sind. ${ }^{27)}$ Genau dieses leistet der vielzitierte erste Satz aus Aristoteles' Peri hermeneias und die daraus entwickelten Definitionen von

23) Bei einer kursiven Verbindung etwa von $m$ und e mag die Grenze zwischen beiden Elementen nicht genau anzugeben sein. Doch irgendwo in einem bestimmten Intervall hört das erste auf und beginnt das zweite, und es liegt kein drittes zwischen ihnen. Die effektive Differenzierung wird hier also durch die Orthographie gewährleistet. Vgl. zu diesem Begriff Goodman 1997, S. 128 ff. - dort spricht Goodman allerdings noch von endlicher Differenzierbarkeit - und Goodman und Elgin 1989, S. $166 \mathrm{ff}$.

24) In der Sprachphilosophie Herders und W. von Humboldts finden sich dagegen durchaus schon Reflexionen, wo dieser Tatbestand berührt wird. Vgl. W. von Humboldt, Ueber die Buchstabenschrift, WW III, S. 89 ff.

25) Vgl. hierzu im einzelnen Stetter 1997, Kap. 8 und 9.

26) Ebd. S. $360 \mathrm{ff}$.

27) Geleistet ist diese Protologik im Kratylos. Vgl. hierzu Lorenz und Mittelstraß 1967 und Stetter 1997, S. 322 ff. 
onoma und rhema. Der etwas von etwas behauptende Satz besteht qua Verknüpfung von genau diesen beiden Elementen, und er ist wahr genau dann, wenn das onoma etwas bezeichnet - semainei $\mathrm{ti}^{28)}$-und wenn dieses so Bezeichnete, die erste Substanz, unter die Extension des rhema fällt.. ${ }^{29}$.

Es ist diese extensionale Semantik, die Aristoteles die Behandlung von logoi als Symbolschemata in Goodmans Sinne ermöglicht, denn unter der Voraussetzung, daß nur (quantifizierte) Namen und Prädikate in Satzschemata vorkommen, kann man formale, vom Sinn der jeweiligen Sätze und ihrer Verknüpfungen abstrahierende Logik betreiben. Dieses logische Fundament gibt der späteren Grammatik ihre interne Gestalt. Seither besteht der Satz aus den nunmehr grammatischen Formen Subjekt und Prädikat - Begriffe, die in der neuen Umgebung auch einen neuen Sinn erhalten: nicht mehr Namen für ontologische Entitäten, sondern für sprachliche Formen.

Mit diesem logischen Ursprung der Grammatik ist gleich in zweifacher Weise eine, wenn man so will, Entrhetorisierung ihres Begriffsrepertoires verbunden ${ }^{30\rangle}$ Zum ersten, unmittelbar, blendet der extensional begründete Wahrheitsbegriff, der der platonisch-aristotelischen Logik zugrunde liegt, den kommunikativen Rahmen aus, innerhalb dessen darum gestritten wird, ob die Verwendung der Namen jeweils sinnvoll, weil den logischen Spielregeln gemäß ist. Das Sprachspiel, das nunmehr gespielt wird, setzt

28) Vgl. Peri hermeneias I, 16a 18, II 16b 21 und passim.

29) Die Eingangsdefinition von Peri hermeneias 16a hat also den schlichten logischen Zweck, den allgemeinen Begriff von Extension zu formulieren, den Aristoteles für seine logische= Semantik braucht. Das pathema tes psyches, das in der christlichen Tradition dann zum conceptus mutiert (vgl. Ockham, Summa Logica I, 1, 2 f.), ist bei Aristoteles nichts als die Extension des sprachlichen Namens. In den Kategorien, Kap. III, ist dies bereits vorausgesetzt. Ohne diese Voraussetzung wäre die im IV. Kapitel der Kategorien getroffene Auszeichnung der ersten ousia als eines Seienden, das niemals selbst ausgesagt werden kann, sondern von dem nur etwas ausgesagt werden kann, undenkbar.

30) Der Begriff der Entrhetorisierung faßt freilich den Vorgang nur aus einer Perspektive, von der nicht ausgemacht ist, daß es die zentrale ist. Ich verwende ihn nicht in systematischer Absicht. 
den für die Philosophie gewonnenen Kratylos oder Phaidros bereits voraus. Zum zweiten, subtiler, wird von Aristoteles mit den Mitteln der neuen technē die Rhetorik selbst als Kunst überzeugenden, weil logischen Argumentierens neu begründet, aus der aller rhetorische Schmuck verbannt wird mit Ausnahme der Metapher. Die wird ihrerseits wiederum mithilfe einer extensionalen Semantik erklärt. ${ }^{31\rangle}$ Die Norm, unter der sich allmählich in der literalen Praxis ein formales Bild von Sprache ausbildet, sind die Gelingensbedingungen der nichtmetaphorischen, behauptenden, an einen anonymen Verstand adressierten Rede. Daß die in der Methodologie der modernen Grammatiktheorie üblichen Beispielsätze, die wir oben schon in anderem Zusammenhang berührt hatten, gleichsam als Zeichen professioneller Seriosität von chronischer Stupidität sind, ${ }^{32}$ hängt mit dieser Herkunft der Grammatik zusammen, der mit dem rhetorischen auch das ästhetische Moment ausgetrieben wurde.

Für unseren Zusammenhang genügt es - die drastischen Abkürzungen seien der Systematik zuliebe gestattet -, zum Beleg der These auf Wilhelm von Humboldts Unterscheidung zweier Sprachkonzeptionen zurückzugehen: die einer Nominalansicht und Pronominalansicht von der Sprache. ${ }^{33}$ Erstmals in unserer Tradition werden hier in einer Philosophie der Grammatik Besonderheit und Beschränktheit des tradierten grammatischen Bildes der Sprache nicht nur gesehen, ${ }^{34}$ sondern einer neuen Ansicht auf sie entgegengesetzt.

Humboldt revidiert die aristotelischen Grundlagen der Grammatik gleich an beiden archimedischen Punkten der tradierten Nominalansicht: Die basale, alle weiteren kognitiven Akte fundierende Referenzfunktion der Rede wird vom Nomen auf das Pronomen verlagert. Grundlage, das jeweils Gemeinte , verständlich' zu artikulieren, ist nicht die Bezugnahme auf die Objekte, um die es geht, sondern die Identifikation von Sprecher und

31) Poetik Kap. 21, 1457 b.

32) Vgl. hierzu Stetter 1997, Kap. 5

33) Grundlegend der Dualis und die Verschiedenheiten des menschlichen Sprachbaus. Vgl. hierzu Bosche 1981, S. 277 ff., di Cesare 1996 und Stetter 1997, S. $491 \mathrm{ff}$.

34) Dies ist zweifellos schon bei Herder der Fall. Man vgl. etwa Teil I, 3 seiner Abhandlung über den Ursprung der Sprache, Frühe Schriften S. $733 \mathrm{ff}$. 
Adressaten der Rede. ${ }^{35)}$ Erste und zweite Person gehen systematisch der dritten voraus. Damit ist die kommunikative Einbettung der Rede in ihren sozialen Kontext, ihre jeweilige Adressierung an jemanden, kategorial dem theoretischen Bild von Sprache wiedergewonnen.

Zum zweiten wird an die Stelle des rhema, des Prädikats, das Verb gesetzt: Zwar erkennt Humboldt die in der Nominalansicht der Sprache geleis tete Parallelführung von logischen und grammatischen Kategorien insoweit an, als seinem Hauptsatz zufolge, der in der Sprache das den Gedanken bildende Organ sieht, jeder Satz als solcher zugleich logische Form sein muß ${ }^{36)}$ Doch sieht er in dieser Parallelführung auch die Differenzen: Die Logik betrachtet den Sachverhalt abstrakt, im „Gebiete“, so Humboldt, "des absoluten Seyns“. ${ }^{37}$ Das Prädikat drückt daher in logischer Hinsicht nicht mehr aus, als daß dieses oder jenes Objekt $\mathrm{x}$ unter den von ihm bezeichneten Begriff y fällt. Dies kategorisch, ohne jede zeitliche oder modale Begrenzung. Das Verb, das in diesem Zusammenhang namentlich als Zeitform relevant wird, leistet demgegenüber etwas ganz anderes: Damit, daß es in der verbalen Performanz aktualiter gesetzt wird, schließt es die verschiedenen referentiellen oder prädikativen Ausdrücke zu einem Ganzen zusammen, einem einheitlichen Symbolschema, und bezieht dieses auf seinen spezifischen Erfüllungsgegenstand - die aktuelle Redesituation, die ihrerseits in der Pronominalstruktur des Satzschemas angedeutet ist. Daher die ,grammatische' Kategorie des Tempus, die in den Verbsystemen des Griechischen oder Lateinischen in besonders hohem Maße formal ausdifferenziert war und die von daher Humboldt das Paradigma seiner Theorie an die Hand gegeben hat.

Die Performanz der Zeit- oder Situationsreferenz kann der Sache nach erst geleistet werden, wenn der Sachverhalt, der in Rede steht, hinreichend deutlich konzipiert ist. Das macht aus dem Verb die hierarchisch höchste Konstituente der Syntax natürlicher Sprachen. Es verdient mehr als nur historisches Interesse, daß Humboldt in diesem Zusammenhang erstmals eine

35) Der Pronominalansicht liegt somit die erstmals im Dualis als ,Urtypus' aller Sprachen beschriebene dialogische Anlage der Rede zugrunde. Vgl. Dualis, WW III, S. 138 ff ff. und Verschiedenheiten, WW III, S. $200 \mathrm{ff}$.

36) Vgl. Grammatischer Bau, GS VI, S. 346 ff., dazu Stetter 1997, S. $491 \mathrm{ff}$.

37) GS VI, S. 346. 
explizite Theorie der Syntax, der „Redeverbindung “ ${ }^{38)}$ entwickelt, in der die Aspekte der Konstituenz und Dependenz systematisch zusammengeführt sind. ${ }^{39)}$

In der Redeverbindung ${ }^{40)}$ sind alle Konstituenten der, Periode' zu einem Gestaltschema zusammengefaßt. ${ }^{41)}$ Dieses verortet Humboldt der scholastischen Psychologie gemäß, die Kants Transzendentalismus zugrunde liegt, im Bewußtsein des Sprechers. ${ }^{42)}$ Doch faßt das Schema als solches eben auch die konkrete Äußerung zu einer Gestalt zusammen, und Humboldt wäre nicht durch die Schule der Kritik der Urteilskraft gegangen, um nicht die ästhetische Dimension zu erkennen, die in diesem Sachverhalt liegt. Als Äußerung ist die Gestalt ergon, damit nicht nur dem rezipierenden Verstand als Objekt gegeben, sondern auch der damit einhergehenden Einbildungskraft. Diese Gestalt wird nun den Verstandesformen, die dem Gesagten inhärent sind, adäquat sein oder auch nicht. Dementsprechend wird die Einbildungskraft, so sie mit Geschmack gepaart ist, diese Redeverbindung als mehr oder weniger gelungen beurteilen. Auf diesem Wege gelangt Humboldt zu einem Begriff der symbolischen Funktion grammatischer Formen, der nun in der Tat völlig quer steht zur logischen Tradition der Grammatik. ${ }^{43}$ Symbolisch kann die grammatische Form deswegen wirken, weil sie in der konkreten Anschauung von Formen dem sprechenden wie rezipierenden Geist Winke gibt, sich mehr bei ihr zu denken, als sie „wörtlich“ ausdrücken kann. ${ }^{44}$ So kann ein gelungener grammatischer Bau einer Sprache - hier denkt Humboldt natürlich an das klassische Griechisch - die Phantasie der diese Sprache Sprechenden beflügeln. ${ }^{45}$ Für einen Moment ist auch die Ästhetik wieder mit dem , grammatischen' Sprachbild versöhnt.

Die Sprachwissenschaft des 19. Jahrhunderts geht an dieser in ihrer Zeit singulären Konzeption vorbei, tut sie als Spekulation $\mathrm{ab}^{46)}$ und wirft sich auf die Erforschung der Diachronie, die ihr in tausend Einzelfakten zerfällt. Die

38) Vgl. Grundzüge, GS V, S. $445 \mathrm{ff}$.

39) Vgl. hierzu Stetter 1997, S. $497 \mathrm{ff}$.

40) Vgl. Grundzüge, GS V, S. $445 \mathrm{ff}$.

41) Vgl. Grammatischer Bau, GS VI, S. 348.

42) Vgl. Grundzüge, GS V, S. 454.

43) Vgl. hierzu Stetter 1997, S. $502 \mathrm{ff}$. 
Gründe dafür können hier nicht erörtert werden. Sprache als Symbolschema oder, in linguistische Terminologie, als System gerät damit aus dem Blick. Eine Revision dieser Perspektive auf den Gegenstand wird erst durch F. de Saussure geleistet, ausgehend vom frühen Mémoire, in der Saussure gegen die junggrammatische Dogmatik die Morphologie der Linguistik als genuinen Gegenstandsbereich zurückgewinnt, ${ }^{47)}$ damit die systematische und zugleich semiologische Perspektive auf die Sache, ${ }^{48}$ über die Skizzen gebliebenen Arbeiten der 90er Jahre bis hin zu den späten cours de linguistique générale. In diesen Vorlesungen, deren Sprengkraft weniger dem Text ihrer Nachschriften als dessen Wirkungsgeschichte ablesbar ist, die ja bis heute anhält, hat Saussure eine Axiomatik der Linguistik entwickelt, die die Gestalt der Sprachtheorie des 20. Jahrhunderts in wesentlichen Zügen geprägt hat.

Leitend sind dabei eine Reihe von Unterscheidungen gewesen, die den logischen Raum umreißen, in der diese Gestalt nunmehr neu situiert wird.

44) Dies wird bei Humboldt folgendermaßen begründet: Wäre eine grammatische Form X nicht mehr als Index der Verknüpfung zweier Ausdrücke A + $\mathrm{B}$, so würde dies, wie er sagt, „zu einem endlosen Einschachtelungssystem führen" (Grammatischer Bau, GS VI, S. 362), da in dem Ausdruck A + X+ B ja nun zwei weitere Verknüpfungsindizes erforderlich wären, um die synthetische Einheit von A + X bzw. X + B auszudrücken usw. ad inf. Die grammatische Form ist also als reines „Verknüpfungszeichen “ mißverstanden. Sie symbolisiert über diese Funktion hinaus die Einheit der Rede als Gestalt. Mit Goodman wäre hier von Exemplifikation zu sprechen. Es würde zu weit führen, den in der Tat schwierigen Gedanken Humboldts von einer symbolischen Funktion der Grammatik in diesem Sinne weiter auszuführen. Dieses Problem führt zum Kern einer Philosophie der Grammatik. Vgl. hierzu auch Stetter 1997, S. 508 ff.

45) Vgl. hierzu Stetter 1997, S. 502 ff.

46) So schon Jacob Grimm, der mit seinem völligen Theorieverzicht dem späteren Positivismus der diachronischen Linguistik des 19. Jahrhunderts zweifellos Vorschub geleistet hat.

47) Vgl. hierzu Stetter 1992, S. 511 ff.

48) Das Morphem ist für Saussure Zeichen: "Le vrai nom de morphologie serait: La theorie des signes - et non des formes ..." (N 7, EC fasc. 4, S. 17). 
Die erste ist die Unterscheidung von Synchronie und Diachronie, Resultat der Sprachwissenschaft des 19. Jahrhunderts. Die zweite ist die von Schrift und Sprache, mit der die Linguistik sich aus der Philologie emanzipiert. Die dritte schließlich ist die von langue und parole, von System und - mit Humboldt zu sprechen - ,jedesmaliger Rede“. Als zentraler Gegenstand der Sprachwissenschaft schält sich in der Rezeption der saussureschen Doktrin mehr und mehr das Studium des Systems heraus. Paradigma einer, strukturalen' Sprachansicht ist die Phonologie der Prager Schule geworden. Sie hält - eindrucksvoll im Werk Roman Jakobsons bezeugt - an Saussures Grundbegriff des signe linguistique fest. Die sprachliche Einheit ist, was sie ist, qua Zeichen: Einheit von signifiant und signifié. Sie vernachlässigt andererseits in der Konzentration auf das System den korrelativen Aspekt der parole, der Performanz. Und gleichermaßen gerät über der Konzentration auf das Studium allgemeiner Eigenschaften von Sprache nach und nach, wenngleich noch nicht programmatisch, dasjenige aus dem Blick, was das Zentrum der Sprachphilosophie Herders und Humboldts ausgemacht hatte: der Aspekt der Verschiedenheit menschlicher Sprachen als eines wesentlichen Charakteristikums menschlicher Sprache. Im Gegensatz zur atomistischen Linguistik der Junggrammatiker konzentriert sich der Blick der Prager Schule ganz auf universelle Aspekte der Sprache. Jakobsons mittlerweile klassische Studie über Kindersprache, Aphasie und allgemeine Lautgesetze ${ }^{49)}$ ist bis heute das vielleicht schönste Dokument dieses Denkansatzes.

Zugrunde liegt diesem die schon bei Saussure eingeleitete Psychologisierung der Sprachtheorie. Von hier aus führt ein direkter Weg zum Mentalismus Chomskys, der sich allerdings in der konzeptionellen Ausgestaltung dieses Modells radikal von Saussures Sprachbegriff wie dem des frühen Strukturalismus unterscheidet. ${ }^{50)}$ Nicht mehr das Sprachsystem ist für ihn Gegenstand der Linguistik, sondern die Grammatik, genauer: die Syntax. Und diese nicht mehr als Symbol- oder Zeichenschema, sondern als im Genom angelegtes Modul. ${ }^{51)}$ Hier geht nun die klassische Schulgrammatik mit einer in Chomskys Arbeiten immer deutlicher zutage tretenden

49) Vgl. Jakobson 1969.

50) Diesen Paradigmenwechsel habe ich in Stetter 1997, S. 229 ff., genauer beschrieben. 
biologischen Konzeption von Sprache einen in der Tat einzigartigen Synkretismus ein: Die höchste Form, die das Syntaxmodul zu generieren imstande ist, ist der Satz, eine Ausdrucksform, die Saussure aus Gründen, wie wir oben bereits betrachtet hatten, der parole zugeordnet hatte. ${ }^{52}$ Seine Hauptkonstituenten - wenn man von den technischen Details einmal absieht - sind Subjekt und Prädikat. Alle logischen wie literalen Voraussetzungen, die - historisch rekonstruierbar - diesem Begriff des Satzes zugrunde liegen, werden mit einer schlichten Setzung als solche gestrichen, ohne daß dies, meines Wissens, in der Theorie der generativen Linguistik auch nur ein einziges Mal thematisiert worden wäre. Die Linguistik - will man das Resultat dieser Entwicklung polemisch auf den Punkt bringen - ist in diesem Projekt als empirische Disziplin auf der Strecke geblieben. Unschwer ist zu sehen, wie dies mit der Vernachlässigung der Performanz als eines theoretisch relevanten Bereichs der Sprachwissenschaft zusammenhängt, mit der hiatischen Trennung von Kompetenz und Performanz, die für den mentalistischen und funktionalistischen Ansatz der generativen Linguistik $^{533}$ konstitutiv ist.

\section{Zwischenbilanz}

Bei all ihren problematischen Aspekten hat dieser Ansatz doch das große Verdienst, die Sprachtheorie zu einem Punkt gebracht zu haben, wo nun der kategoriale Raum deutlich wird, in dem eine nach-chomskysche Sprachtheorie anzusiedeln ist.

Hinter die Auffassung, daß allen sprachlichen Performanzen, all dem, was immer man in empirischem Sinn als "menschliche Sprache" bezeichnet, eine den Menschen charakterisierende Sprachfähigkeit zugrunde liegt und daß dieser Gesichtspunkt in der Gestalt der Sprachtheorie angemessen zur Geltung zu bringen ist, wird man kaum zurück können. Doch dessen ungeachtet ist ein Mentalismus chomskyscher Prägung zweifellos kein gangbarer Weg. Zu dieser Konsequenz zwingt - neben vielen weiteren

51) Vgl. zur wissenschaftstheoretischen Charakterisierung dieses Modells i. e. Schneider 1992, S. $41 \mathrm{ff}$.

52) Vgl. CLG S. $172 \mathrm{f}$.

53) Vgl. hierzu auch Putnam 1991. 
Argumenten - insbesondere die oben beschriebene Käfer-in-derSchachtel-Aporie.

Die faculté du langage hat man logisch als distributives Allgemeines $\mathrm{zu}$ konstruieren: Sie ist zur genomischen Ausstattung jedes menschlichen Individuums zu rechnen. Ihre Beschreibung fällt damit in den Bereich der Neurobiologie..$^{54}$ Doch ebenso eindeutig hat man sich die Sprachfähigkeit als Fähigkeit der Ausbildung von Sprachen qua Zeichen- oder Symbolsysteme zu denken, und die einzig uns zugänglichen natürlichsprachlichen Symbolsysteme sind eben Sprachen, nicht die Sprache. Gerade wenn man in der faculté du langage die eine, jeder Erscheinungsform menschlicher Sprache zugrunde liegende Basis erkennt, so schließt man damit eo ipso universalsprachliche oder universalgrammatische Konstrukte als Erklärungsgrundlage der Vielfalt empirischer sprachlicher Formen aus. Alle solche Konstrukte können - aus rein logischen Gründen - nie etwas anderes sein als ungedeckte Hochrechnungen aus besonderen einzelsprachlichen Sprachverwendungen. Chomsky hat - in seinem systematisch doppeldeutigen Gebrauch des Wortes "Grammatik“ - Namen für Elemente einer Universalgrammatik dadurch gebildet und damit ein logisches Verständnis der Rede über diese Gegenstände ermöglicht, daß er die Extension bestimmter, uns aus unserer grammatischen Tradition geläufiger Namen programmatisch verdoppelt, aus dieser Verdoppelung aber gleichzeitig das Besondere gestrichen und das so gewonnene Konstrukt als empirisch gegeben vorausgesetzt hat: Der Ausdruck der Hans ist Subjekt des Satzes der Hans liebt die Inge, und gleichzeitig soll der Name "Subjekt" Bezeichnung einer Form der Universalgrammatik sein. Doch damit wird dem Namen "Subjekt" im zweiten Glied dieser unschuldigen Konjunktion ein, mit Frege zu sprechen, anderer Sinn gegeben als in der Verwendung des ersten Gliedes, ein Sinn, der in keinem Beispiel derselben Form auch nur angedeutet werden könnte. ${ }^{55}$

Die hier sichtbar werdende Paradoxie kann man am besten vielleicht folgendermaßen fassen: Indem Chomsky die Grammatik als privatsprachliches Konstrukt konzipiert hat, hat er nolens volens gezeigt, daß diese Konzeption unhaltbar ist. ${ }^{56}$ Jede Sprachtheorie muß - dies ist die Lehre, die aus den vielfältigen Wandlungen des Chomsky-Paradigmas zu ziehen sind -

54) Vgl. hierzu etwa Spitzer 1996. 
Sprache als ,öffentliche' Sprache rekonstruieren.

Öffentliche Sprachen aber sind Zeichen- oder Symbolsysteme - wie immer man Sprache ansonsten theoretisch fassen wird. Dieses Verständnis von Sprache geht jeder Sprachtheorie voraus - auch der chomskyschen, die ja in der Architektur ihres Modells selbst auf der Ebene des Syntax-Moduls nicht vollständig davon abstrahieren kann, daß irgendwie und -wo den dort beschriebenen Formen eine wie auch immer geartete Bedeutung ,zuzuordnen “ ist. Damit kommt neben der Neurobiologie als zweite mit dem Gegenstand Sprache befaßte Disziplin nun die Psychologie ins Spiel, denn Bedeutung welcher Art auch immer - ob wir nun von Wort-, Satz- oder Ausdrucksbedeutung sprechen - ist stets Resultat eines Prozesses, in dem aktualiter uno actu Zeichen- oder Symbolketten gebildet und, mit Goodman zu sprechen, auf Bezugnahmegebiete bezogen werden. Zum Symbol oder in linguistischer Terminologie - zum Zeichen wird der Ausdruck erst dadurch, daß er als Einheit verstanden wird. Diese Einheit kann - das hat in der gesamten Tradition der Sprachtheorie am klarsten wohl W. von Humboldt gesehen - nur eine "gedachte“, in moderner Terminologie, eine kognitiv erzeugte sein.

Selbst dies noch kann man der formalen Gestalt abgewinnen, die Chomsky der Sprachtheorie gegeben hat. Liest man eine Phrasenstrukturgrammatik als Beschreibung eines Satzgenerierungsprozesses, so hat die Abfolge von Ersetzungsregeln genau den Sinn, daß eine Einheit in Konstituenten zerlegt oder, umgekehrt betrachtet, aus ihnen aufgebaut wird. Zerlegen kann man aber - in saussurescher Terminologie - nur den signifiant, nie den signifié, denn dieser ist eben gedachter Natur. Man zerlegt nicht das Wort ,in“, indem man es in Teile „i“ und "n“ zerlegt, sondern dann zerlegt man den Ausdruck. Das Wort selbst ist als Einheit von

55) In jedem beliebigen Beispiel würde ja der Ausdruck „Subjekt-von“ nach Bedingungen, die völlig unabhängig von jeder Theorie einer Universalgrammatik sind, auf einen einzelsprachlichen Ausdruck als seine Extension bezogen werden, und dann hängt es eben nur von dem jeweiligen besonderen Verständnis von Grammatik und von der jeweiligen Sprache ab, ob dieser bestimmte Ausdruck zurecht als Subjekt dieses bestimmten Satzes aufgefaßt wurde.

56) Vgl. hierzu i. e. Stetter 1999. 
signifiant und signifié weder in diese noch in andere Konstituenten teilbar. ${ }^{57}$ Analoges gilt für jeden komplexen sprachlichen Ausdruck, dem wir , einen' bestimmten Sinn zusprechen. ${ }^{58}$ Morphologie und Syntax natürlicher Sprachen müssen in diesem Sinn als Artikulationssysteme von Sinneinheiten aufgefaßt werden. ${ }^{59}$ )

Als dritter Kernpunkt, der aus der oben skizzenhaft durchgeführten Revision der Geschichte der Sprachtheorie festzuhalten ist, bleibt das Verhältnis von Sprache und Schrift festzuhalten, damit die Frage von analoger und digitaler Gestaltung der sprachlichen Performanz. Dies ist eine historische wie systematische Frage. Die moderne Sprachtheorie kann nicht wie dies in der generativen Linguistik bis heute der Fall ist - das Faktum vernachlässigen, daß zu den Voraussetzungen von Sprachtheorie konstitutiv gehört, daß uns Sprache als orale wie als literale wie - so wäre hinzuzufügen als Gebärdensprache "gegeben“ ist. Alle drei Formen sind Produkte der Evolution und als solche Produkte der menschlichen faculté du langage. Als wissenschaftlicher Gegenstand wird Sprache jedoch nur über den Weg der Verschriftung erfahrbar, ${ }^{60)}$ und somit stellen sich die Fragen einer analog oder digital angelegten Phonologie, Graphematik, Morphologie, Syntax und

57) Die Paradoxien, die sich hieraus für ein philosophisches Verständnis von Sprachtheorie ergeben, habe ich am Fall des saussureschen Arbitraritätsprinzips in Stetter 1997, Kap. 4, diskutiert.

58) Eine entsprechende semiologische oder semiotische Interpretation der Ersetzungsregel habe ich in Stetter 2001 entwickelt.

59) Chomskys Konzept einer, autonomen' Syntax ist - wenn man es seiner mentalistischen Programmatik entkleidet - am Modell symbolischer bzw. maschineller Sprachen entwickelt worden. Dort hat es zweifellos seinen guten Sinn. Eine Philosophie der Sprachtheorie muß aber in diesem Punkt darauf insistieren, daß diese Analogie irreführend ist, wenn sie der "Architektur" dieser Theorie zugrunde gelegt wird. Es ist ja auch unschwer zu sehen, daß die Rede von syntaktischen ,Formativen', die von einer anderen Ebene her semantisch oder phonologisch interpretiert werden, hinfällig wird, sobald man sie wörtlich nimmt. Die theoretische Crux der Theorie Chomskys ergibt sich aus der faktischen Informalität der Theorie.

60) Vgl. hierzu Stetter 1997, Kap. 3. 
Semantik schon auf dieser ersten Ebene einer linguistischen Phänomenologie.

\section{Konsequenzen: die Natur des sprachlichen Zeichens}

Als erste Konsequenz ergibt sich aus dieser Bestandsaufnahme, daß der Gegenstand Sprache ein interdisziplinärer Gegenstand ist. In dem hier betrachteten Zusammenhang ist vor allem die Grenzziehung zwischen den Nachbardisziplinen Linguistik und Psychologie von Interesse. Zweifellos stehen sie im Zentrum eines Forschungszusammenhangs, der die Brücke von der Ebene der faculté du langage bis hin zu dem des Studiums der Formen bestimmter sprachlicher Erscheinungsformen zu schlagen hat.

Was beide Disziplinen zusammenbringt, ist die Auffassung, daß sprachliche Einheiten Symbolschemata in dem von Goodman beschriebenen Sinne sind. Saussures Begriff des signe linguistique als Einheit von signifiant und signifié war unzureichend in genau dem Punkt, daß in ihm von der Referenz abgesehen war. ${ }^{61)}$ So verständlich dieser Ansatz bei Saussure auch in seiner Wendung gegen Auffassungen gewesen ist, die in der Sprache nichts anderes sahen als eine Nomenklatur ${ }^{62)}$ - er hat den Begriff des sprachlichen Zeichens doch mit einer Hypothek belastet, die die theoretische Vernachlässigung der Performanz zur Folge gehabt hat. Das aus Saussures Semiologie resultierende Konzept des Wertes, den ein sprachlicher Term im Systemzusammenhang repräsentiert, muß indessen für die Sprachtheorie grundlegend bleiben oder, genauer, ihr wieder zugrunde gelegt werden. ${ }^{63)}$ Aus ihm folgt das systematische Argument gegen die Aufteilung einer wie auch immer gearteten mentalen Grammatik in Kern und Peripherie: Jedes Element im System ist, was es ist, je nur in Korrelation zu ähnlichen und unähnlichen im gesamten System. Deswegen ist es unlogisch, beispielsweise - im System des Lateinischen - den Akkusativ als universellen Kasus gegenüber dem Ablativ als peripherem auszuzeichnen. Beide

61) Vgl. hierzu Stetter 1997, S. $214 \mathrm{ff}$.

62) Vgl. hierzu die frühen notes $\mathrm{N} 12$ und vor allem die notes item N 15, EC fasc. 4, S. $35 \mathrm{ff}$.

63) Ich verzichte hier darauf, Saussures Argumente im einzelnen zu rekapitulieren. Zur Systematik vgl. Stetter 1997, S. 208 ff. 
gehören als Kasus zu einem Subsystem, und aufgrund dieser besonderen Korrelation hat der Akkusativ im Latein einen anderen Wert als der im Griechischen, weil letzteres weniger Kasus hat als das Latein, dort sich die Funktionen eben anders auf die Systemelemente verteilen. ${ }^{64)}$

Jedes sprachliche System muß daher erstens als in sich homogenes und zweitens als ein Ganzes begriffen werden. Wiederum hat dies wohl am klarsten W. von Humboldt gesehen, wenn er in den Grundzügen des allgemeinen Sprachtypus von, dem' Verfahren der Sprache spricht, qua Artikulation von Wörtern und "Perioden“ die Rede zu bilden. ${ }^{65}$ ) Auf jeder Ebene bilden deren Einheiten homogene Systeme, und für die Syntax als das hierarchisch höchste dieser Systeme muß das in gleicher Weise gelten. Insofern ist jede individuelle faculté du langage als Fähigkeit zu begreifen, eine Sprache als ein solches ganzheitliches, homogenes System auszubilden. Erklären können wird man - soweit Erklären-warum hier überhaupt möglich ist - diese Fähigkeit nur im Verbund mit Psychologie und Neurobiologie. Für die Linguistik als Spezialdisziplin ist die Forderung explanativer Adäquatheit zweifellos nicht einlösbar. Hier ist - jedenfalls in erster Instanz - zunächst einmal empirisch triftige Phänomenologie gefordert.

Eine sprachliche Einheit gewinnt Bedeutung erst im Gebrauch. Auch hinter diese Einsicht wird eine nach-chomskysche Linguistik nicht zurückgehen können. Versuche funktionalistischer Deutung bestimmter grammatischer Formen sind andererseits immer wieder gescheitert. Insofern bleibt nur. der Weg, auf jegliche intensionale Charakterisierung der Weisen zu verzichten, in denen die Verwendung sprachlicher Ausdrücke deren Bedeutung prägt, und es extensional bei dem puren Faktum der

64) Unlogisch ist die Rede von universellen Elementen im Unterschied zu peripheren, weil sie auf einer Homonymie beruht. Man macht sich nicht klar, daß das Symbolschema „Kasus“ auf verschiedene Bezugnahmegebiete angewandt wird, wo zudem die Verifikationsbedingungen hinsichtlich der Frage, was in diesem nun Erfüllungsgegenstand des Schemas ist, unvergleichbar sind. Also wird das Schema äquivok verwendet.

65) Sein Terminus für Syntax ist daher auch "Redeverbindung“, vgl. Grundzüge $\S \S 127$ ff., GS V, S. 445 ff.; vgl. zum Gesamtzusammenhang Grundzüge $\S \S 56 \mathrm{ff} ., \mathrm{GS}$ V, S. $400 \mathrm{ff}$. 
Bezugnahme von sprachlichen Schemata auf etwas zu belassen. Eben dieser Gedanke zeichnet die Symboltheorie Goodmans aus.

Was dem Schema - Wort, Phrase, Satz, ... - Bedeutung gibt, ist danach zweierlei: erstens sind es die syntaktischen Bezüge, in die es in der Performanz gestellt wird, zweitens, semantisch, die Zuordnung von Erfüllungsgegenständen zu den entsprechenden Schemata, die durch die Verknüpfung von indexikalischen und prädikativen Subschemata geleistet wird. ${ }^{66)}$ Was - um hier nur den einfachsten Fall zu nehmen - dem Wort im Satzzusammenhang Bedeutung gibt, ist also erstens seine Bezugnahme auf den syntaktischen Kontext und zweitens die auf das nichtsprachliche Bezugnahmegebiet: die Performanzsituation, die Adressierung, die Objekte, über die gesprochen wird, usw. Diese Bezugnahmen sind kognitive Leistungen. Auch das, was Saussure als den Wert eines Terms im Rahmen dies Sprachsystems bezeichnet hat, kann nur mithilfe derartiger Bezugnahmeleistungen rekonstruiert werden. ${ }^{67}$ Erfüllungsgegenstände der Abbildung eines Verbs auf die Menge koexistierender Terme wären dann in syntaktischer Hinsicht etwa Kasusmerkmale, in semantischer Hinsicht z. B. semantische Implikationen, die mit Lexemen verbunden sind usw. Was also den syntaktischen wie semantischen Wert eines sprachlichen Symbolschemas, $z$. B. eines Wortes ausmachte, wäre danach eine bestimmte Menge von syntaktischen und semantischen Bezugnahmeleistungen $\left\{B_{\text {syn } 1}\right.$,

66) Ich belasse es hier bei diesen zwei Hauptkategorien. Jede empirische Grammatik weist besondere Mischformen auf. Dies ist eine Frage, die zu diskutieren ist, wenn es darum geht, ob sprachliche Schemata in syntaktischer und semantischer Hinsicht digital oder analog organisiert sind.

67) Erfüllungsgegenstände des Terms A eines Systems S sind jeweils bestimmte Terme B, C, ..., I desselben Systems oder - umgekehrt betrachtet - das System ist genau hierin eines, daß seine Terme wechselseitig die Funktionen von Schemata und Erfüllungsgegenständen ausüben. Bei der üblichen Darstellung von syntagmatischen oder paradigmatischen Relationen macht man de facto davon Gebrauch, wobei aber die Suggestion der schriftlichen Darstellung verhindert hat, daß man derartige Repräsentationen ihrerseits symboltheoretisch interpretiert hätte. Alle, selbst die in der formalen Linguistik entwickelten Repräsentationssysteme sind bis heute in dieser Hinsicht logisches Niemandsland. 
$\left.B_{\mathrm{syn} 2}, \ldots, B_{\mathrm{synm}} ; B_{\mathrm{sem} 1}, B_{\mathrm{sem} 2}, \ldots, B_{\mathrm{semn}}\right\}$, die assoziativ mit dem jeweiligen Wortschema verbunden ist, die psychische Matrix des Wortes. Die saussuresche Formel, nach der das signe linguistique als Einheit von signifiant und signifié zu begreifen ist, wäre also zu ersetzen durch das Konzept eines sprachlichen Symbolschemas, in dem ein sprachlicher signifiant aufgefaßt wird als Resultat psychischer Prozesse, durch die er erzeugt und mit einer derartigen Matrix verknüpft wird. ${ }^{68)}$

Linguistik und Psychologie betrachten, nimmt man es genau, die sprachliche Performanz von zwei Seiten: die Linguistik von der des ephemeren Resultats, die Psychologie von der des mentalen Erzeugungsprozesses. ${ }^{69)}$ Was die letztere beschreibt, ist noch nicht sprachlicher Ausdruck, und was die erstere beschreibt, ist nicht mehr kognitiver Prozeß. Der kategoriale Apparat beider Disziplinen muß daher verschieden sein. Das proton pseudos, das die Vorstellung von der Linguistik als kognitiver Disziplin beherrscht hat, ist rückblickend also genau der doppeldeutige Gebrauch des Wortes "Grammatik“ gewesen, mit dem Chomsky in den Aspects die kognitive Wende der Linguistik eingeleitet hat.

Die erste Aufgabe der Linguistik muß daher die genauest mögliche Beschreibung dieses ephemeren Resultats sein, und eine Phänomenologie dieses primären linguistischen Objekts hat die von der generativen Linguistik sträflich vernachlässigte Performanz zu rehabilitieren. Denn dieses Objekt ist ein Performanzphänomen. Die zweite Revision des aktuellen linguistischen Paradigmas, die überfällig ist, wird darin bestehen müssen; von Anfang an verschiedene mediale Ausprägungen von Sprache als Sprachformen gleichen Rechts anzuerkennen, auch wenn phylo- wie ontogenetisch der oralen und der Gebärdensprache zweifellos Priorität vor der Schriftsprache zukommt. Umgekehrt ist diese - erkenntnistheoretisch

68) Diese Matrix beruht ihrerseits auf von der Neurobiologie beschriebenen Sachverhalten (vgl. Spitzer 1996), doch dies kann im folgenden ausgeblendet bleiben.

69) Der psychische Prozeß, der die Sprachproduktion leitet, ruht seinerseits auf neurobiologischen Grundlagen auf. Diesen Aspekt verfolge ich hier nicht weiter, obwohl auch die Erhellung dieser Zusammenhänge zweifellos mit zum Gesamtkomplex dessen gehört, was der Begriff der Sprachfähigkeit umfaßt. 
betrachtet - ebenso zweifellos dasjenige Medium, in dem die anderen beiden medialen Sprachformen repräsentiert sein müssen, um überhaupt linguistischer Gegenstand werden zu können. ${ }^{70)}$ Zudem gewinnt jedes Schriftsystem, je länger es in Kohabitation mit oralen Dialekten lebt, desto größere Autonomie diesen gegenüber. Die Umrisse der Evolution von Schriften, die hieraus erwächst, sind ja von A. Leroi-Gourhan eindrucksvoll beschrieben worden. ${ }^{71}$

In diesem Zusammenhang ist nun in buchstäblichem Sinn von einer Re-Rhetorisierung der Sprachtheorie zu reden: Der erste linguistische Gegenstand ist sich bewegende Rede oder Geste. Die Performanz ist ihr ausschließlicher Seinsmodus. Demgegenüber ist die schriftliche Sprache ebenso ausschließlich starres Resultat einer wie auch immer vermittelten Bewegung. ${ }^{72)}$ Das orale Wort existiert solange und nur solange, wie es gesprochen wird. Das schriftliche beginnt zu existieren, wenn es geschrieben ist. Bei aller Schriftkritik Saussures ist das Bild der langue, des Sprachsystems, das die Linguistik des 20. Jahrhunderts entwirft, doch schon bei Saussure selbst ein eher der Schrift gleichendes: die im Moment der Synchronie stillgestellte Fluktuanz. Die generative Linguistik zeichnet schon von ihrer Methodologie her ein durch und durch schriftgeprägtes Bild der Sprache ${ }^{73)}$ Die Theorie der Universalgrammatik ist eigentlich nichts anderes als die Hypertrophie dieses von einem digitalen Schriftsystem her entworfenen Sprachbildes. Dieses Bild ist genau darin eine Fiktion, daß es die eigenen Konstitutionsbedingungen nicht in Rechnung stellt. Selbst die psychische Matrix noch, die schriftliche Wörter oder Texte erzeugt, ist eine solche, die Bewegung produziert, nicht Starre. Der Text wird, die Textur ist geschrieben. ${ }^{74}$

Also müssen schon die Erzeugungsmodi des oralen von dem des geschriebenen Textes kategorial verschieden sein. Die syntaktischen Bezugnahmen beim Verfertigen der oralen oder der Gebärdenrede beziehen sich stets auf Vergangenes, nur zu Erinnerndes. Die, die das Schreiben

70) Vgl. hierzu Stetter 1997, Kap. 3.

71) Vgl. Leroi-Gourhan 1984.

72) Vgl. hierzu Stetter 2002a.

73) Vgl. hierzu Stetter 1997, S. $260 \mathrm{ff}$.

74) Vgl. ebd. Kap. 7. 
lenken, auf zugleich visuell Präsentes. Im Gegensatz dazu ist der Bezugnahmerahmen der oralen Rede die je präsente Situation, die in der Regel dialogisch ist. Der des Schreibens ist die monologische Situation, in der man sich, wenn überhaupt, an einen imaginierten Adressaten wendet, also aufs Gedächtnis zugreift, nicht auf die Wahrnehmung, und wo die Objekte, auf die man sich bezieht, entweder im Text bereits angelegt, also symbolisch repräsentiert sind, oder aber wiederum durch Gedächtnisleitungen erzeugte. Die logischen Formen, die aus derart unterschiedlichen Produktionsbedingungen resultieren, müssen grundverschieden sein. Dies ist eines der unbezweifelbaren Resultate der wittgensteinschen Diskussion des Privatsprachenproblems. ${ }^{75)}$ Also muß die Ebene der Konstitution logischer Formen von der sprachlicher Formen unterschieden werden. Das macht die Liaison, die die generative Linguistik mit der Tradition des Satz-Begriffs eingegangen ist, unhaltbar.

\section{Essentials einer nach-chomskyschen Sprachtheorie}

Zu den Kernpunkten einer Revision der Sprachtheorie gehört darum der Abschied von dem Dogma, daß der Satz die höchste Kategorie der Syntax sei. Von den Metaregeln einer Phrasenstrukturgrammatik her betrachtet war der dogmatische Charakter dieser Annahme von Anfang an klar. ${ }^{76)}$ An die Stelle der Theorie, die - wie Chomsky zurecht bemerkt hat - das Anfangssymbol zu liefern hätte, ist die dem Bereich symbolischer Sprachen entlehnte Annahme gesetzt worden, daß eine Grammatik als ein Entscheidungsverfahren über der Menge wohlgeformter Sätze aufzufassen sei. Hier tritt die Alphabetinduziertheit des gesamten Ansatzes in besonderer Deutlichkeit zutage. Denn diese Konzeption setzt ja ein digitales Medium voraus, in dem gewährleistet ist, daß die Mengen von Erfüllungsgegenständen, die je zwei verschiedene gleichrangige Kategoriensymbole A und $B$ definieren, welche durch eine Ersetzungsregeln $X \rightarrow A+B$ gegeben sind, die von Goodman beschriebenen semantischen Erfordernisse der Disjunktheit und effektiven Differenzierung genügen. ${ }^{77)}$ Anders wäre das

75) Vgl. Philosophische Untersuchungen I, 243 ff., dazu Stetter 1997, S. 571 ff.

76) Der ersten Metaregel zufolge ist die linke Stelle der ersten Ersetzungsregel ja leer. 
Verfahren kein Entscheidungsverfahren. Doch damit wird der Blick auf das Phänomen verstellt. In jeder Morphologie etwa wird die Unterscheidung von ein- und zweisilbigen Wörtern eine Rolle spielen. Nun nehme man das englische Wort little und suche zu entscheiden, ob es ein- oder zweisilbig ist. Und unter semantischem Gesichtspunkt wird es in jedem natürlichsprachlichen System echte Kopien voneinander geben, z. B. auf Grund und aufgrund, die in syntaktischer Hinsicht keine Kopien voneinander sind. Auf jeder linguistischen Beschreibungsebene wird man Phänomene finden, wo beide Kriterien verletzt werden. Dies liegt am Fluktuanz-Charakter der Sprache ${ }^{78)}$ der doch etwas damit zu tun haben muß, daß nicht nur die Performanz, sondern das System selbst als ein ständig in Bewegung sich befindendes begriffen werden muß. Der Wert eines jeden Terms muß - wie wir gesehen haben - durch Bezugnahmehandlungen aufgebaut werden, in denen die Terme des Systems wechselseitig als Schemata und als Erfüllungsgegenstände fungieren. Dies ist ein Prozeß, der anhält, solange sich ein Sprecher seiner Sprache noch irgend bedient.

Letztlich muß dann für jede beliebige sprachliche Kategorie gelten, daß ihre Grenzen fließend sind. Insofern wäre hier durchaus Saussure in seiner Schriftkritik zu folgen. Die Sprachtheorie muß von einer Phänomenologie des Oralen oder auch der Gebärdensprache ausgehen, d. h. vom Studium entsprechender Performanzen. In erster Instanz muß so Sprachkompetenz als die Fähigkeit zur Erzeugung bewegter Symbolschemata gefaßt werden, die im Fall der oralen Sprache vom Ohr, im Fall der Gebärdensprache vom Auge kontrolliert wird.$^{79}$ Erst in zweiter Instanz wird man dagegen an eine Beschreibung von literaler Sprachkompetenz gehen können. Zusammengenommen mit den Überlegungen zur Natur des sprachlichen Zeichens heißt dies, daß die Annahme einer syntaktischen Tiefenstruktur als Kernbestand der Sprachkompeten $z$ ebenso aufgegeben werden muß wie die Annahme einer Universalgrammatik. Jedes Kompetenz-Modell wird auf

77) Auf den engen Zusammenhang von effektiver Differenzierung eines Symbolschemas und dessen digitaler Qualität hat Goodman verschiedentlich hingewiesen. Vgl. etwa Goodman und Elgin 1989, S. 168.

78) Vgl. hierzu Stetter 1997, S. $126 \mathrm{ff}$.

79) Die Gebärdensprache muß hier aus systematischen Gründen berücksichtigt werden. Weiter gehe ich im Folgenden nicht auf sie ein. 
einer Theorie der , konkreten' Einheiten der Sprache aufbauen müssen. Dies heißt wiederum, daß diese Theorie nicht medienneutral konzipiert werden kann. Die natürliche Sprache existiert in ihren fluktuierenden einzelsprachlichen Ausdifferenzierungen, die bis auf die Ebene von lokalen Dialekten und Idiolekten hinuntergeht. Die logische Grundanforderung an die Sprachtheorie wird folglich darin bestehen, daß sie zu zeigen hat, wie dies unter der Annahme der Universalität der faculté du langage möglich ist.

Geht man dergestalt von der Performanz aus, so lautet die zentrale Frage, die dann zu beantworten ist: Wo ist hier beim Studium sprachlicher Phänomene die Grenze zu ziehen zwischen dem, was in der Performanz genuin sprachlicher Natur ist und dem, was auf mentalen bzw. kognitiven Leistungen anderer Art beruht?

Diese Frage zielt im wesentlichen auf das Kategorieninventar der Syntax. Niemand zweifelt daran, daß die Beherrschung der Regularitäten der Wortbildung einer Sprache und die Fähigkeit zu Veränderungen im Wortbestand selbst wie etwa Neubildungen zum Kernbestand jeder , natürlichen' Sprachkompetenz zählen. So gibt es auch auf der Ebene der Syntax in jeder Sprache eine Fülle von Regularitäten, die man zu diesem Kernbestand rechnen wird.

Nicht einmal auf den Bereich der Bildung von - in Goodman'schen Termen - Symbolschemata, also auf den Bereich der Syntax, wird man diesen Bestand beschränken können. Denn zweifellos zählt mit zur Sprachkompeten $z$ auch die Fähigkeit, Spracheinheiten in Situationen anwenden zu können. Die Frage lautet also, wo die Grenze zu ziehen ist zwischen dem, was als allgemeine sprachliche Regularität in sprachlichen Performanzen auszumachen ist, und dem, was darin als Resultat besonderer kognitiver Leistungen anzusprechen ist, die auf das Konto von logischer, rhetorischer oder ästhetischer Kompetenz zu buchen sind. ${ }^{80}$ ?

Paradigmatisch mag es hier genügen, die Frage darauf zu beschränken, ob die Kategorie , Satz' zurecht als hierarchisch höchste Kategorie der Syntax gehandelt wird. Die Frage ist eben nicht, ob das, was man - aus welchen Gründen auch immer - als Satz bezeichnet, eine sprachliche Grundform darstellt. Sie lautet umgekehrt, ob alles, was überhaupt als syntaktische Grundform beschrieben ist, in letzter Instanz auf eine Kategorie Satz zurückzuführen ist, oder ob an die Stelle des Fragezeichens, das "zunächst", stets auf der linken Seite der ersten Ersetzungsregel einer 
Phrasenstrukturgrammatik anzusetzen ist, vielleicht nicht besser ein Platzhalter für die Elemente einer offenen Menge von Kategoriensymbolen zu setzen wäre, die wir mit dem allgemeinen Begriff der Phrase charakterisieren könnten - das Material, in Humboldts Terminologie das ergon, das der Einbildungskraft oder Phantasie oder Kreativität als endlicher Bestand gegeben ist, damit sie damit "unendlichen“, will sagen nicht in einem Algorithmus zu fassenden Gebrauch macht.

In dieser Hinsicht ließe sich an eine reiche Forschung anknüpfen, in der allgemeine Eigenschaften von Phrasen beschrieben worden sind. Als die zentrale gilt die von "Kopf “ und "Mitspieler“, wobei allerdings die Definitionskriterien durchaus umstritten sind. Als relativ unstrittige Beispiele für derart hierarchisch organisierte Phrasen gelten - zumindest für indoeuropäische Sprachen - Verbal-, Nominal-, Präpositional- und Adjektivphrase. In diesem Begriff der Phrase werden die Prinzipien der Konstituenz und Dependenz zusammengeführt. Dies sind allgemeine Prinzipien jeder Syntax. Von Syntax kann man jedoch nur reden, wo faktische Verbindungen von Wörtern zu umfassenderen Einheiten vorliegen. Also bezeichnet der Begriff der Phrase einen Bereich in jeder Performanz, der von dem bestimmt ist, was man als sprachliche Regularität ansprechen kann. ${ }^{817}$

$\mathrm{Da}$ dagegen eine Phrase stets aus Subjekt und Prädikat bestehen muß, ist weder aus dem Begriff der Konstituenz noch dem der Dependenz abzuleiten. Dies folgt erst aus besonderen Gelingensbedingungen für bestimmte Phrasen, und die sind klarerweise logischer Natur. Allgemein bezeichnet die Illokution in Austins Sinn diejenige Ebene, wo - wie Austin dies beschreibt - von Einheiten der langue ein bestimmter Gebrauch gemacht wird, sodaß sich daraus diese oder jene Äußerung ergibt, die dann eben nicht nur, wohlgeformt' in der Hinsicht ist, daß man sie als Beispiel für be-

80) Diese Grenzziehung hat viele Ähnlichkeiten mit der bekannten Unterscheidung von sprachlichem und Weltwissen. Ich greife diese jedoch nicht auf, weil sie mit dem Begriff des Wissens eine hier nicht angemessene und darum vieldeutige Kategorie ins Spiel bringt. Sprachkompetenz ist nur dann als Wissen anzusprechen, wenn man aus diesem Begriff alle die Konnotationen streicht, die Wissen $z u$ Wissen machen. Vgl. hierzu grundlegend Schneider 1992, S. 64 ff. 
stimmte grammatische Besonderheiten der Sprache X nehmen kann - wie dieser Satz als ein Beispiel für die Möglichkeiten genommen werden kann, im Deutschen eine ungeahnte Anzahl von Nebensätzen aneinanderzureihen sondern auch in der Hinsicht, daß die betreffende grammatische Fügung auch in logischer oder rhetorischer Hinsicht als ,gelungen' gelten kann. $\mathrm{Daß}$ jeder Subjekt-Prädikat-Satz derartige zusätzliche logische Bedingungen erfüllt, ist unschwer zu sehen. Wie sollte es bei der historisch rekonstruierbaren Tradition dieses Konzepts auch anders sein. ${ }^{82}$ Jeder Satz ist eine Phrase, und zwar eine Verbalphrase, doch nicht alle Phrasen sind Konstituenten von Sätzen.

Diese Unterscheidung markiert die Grenze zwischen dem, was man im engeren Sinn als Produktionen von Sprachkompetenz ansehen kann, und dem, was auf das Konto von logischer, rhetorischer oder ästhetischer Kompetenz zu buchen ist. Die abschließend noch zu behandelnde Frage lautet, ob diese Grenze - wie Chomsky dies angenommen hatte - eine strikte ist, sodaß sie in einer Phrasenstrukturgrammatik dargestellt werden könnte, deren Kategorienbestand die oben beschriebenen syntaktischen und semantischen Eigenschaften der Disjunktheit und effektiven Differenziertheit aufweist, oder ob sie fließend ist. ${ }^{83\rangle}$ Weisen syntaktische Kategorien diese Eigenschaften nicht auf, so kann die Grenzziehung nicht strikt sein.

Unter den Phrasen nimmt traditionell die Verbalphrase eine Sonderrolle ein. Sie beruht darauf, daß das finite Verb selbst nie zu einer Phrase

81) Daß mit dem Begriff der Phrase in der Tat schon ein Performanz-Bereich angesprochen ist, ergibt sich aus der logischen Natur der Dependenz. Diese ist ja so geartet, daß stets mit einer bestimmten sprachlichen Einheit, z. B. einem bestimmten Verb wie schenken, bestimmte allgemeine Charakteristika verbunden werden, in diesem Fall, daß das Verb schenken regelmäßig mit drei Ergänzungen im Nominativ, Dativ und Akkusativ verbunden wird. Also setzt Dependenz stets die Wahl eines bestimmten Elements voraus. Vgl. zur genaueren Analyse dieses Problems Stetter 2001, S. $258 \mathrm{ff}$.

82) Vgl. hierzu Stetter 1997, S. 382 ff.

83) Logisch ist anzumerken, daß die Frage der Universalgrammatik mit dieser Unterscheidung steht und fällt. 
expandiert werden kann. Als nicht phrasenbildend gelten andererseits die Personalpronomen, die jedoch Hauptkonstituenten von Sätzen bzw. Verbalphrasen sein können. Man hat also bereits an der zweithöchsten syn-

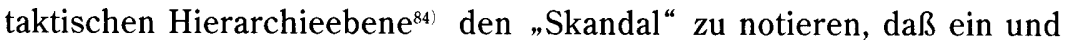
dieselbe Kategorie - Subjekt- oder Objektergänzung z. B. - phrasenfähig und nicht phrasenfähig sein kann und damit in sich dieselbe Spannweite an verschiedenartigen Erscheinungsformen aufweist wie die Syntax insgesamt. Für die Endelemente der Syntax gilt dies nicht. Je hierarchisch höherrangig, so muß man folgern, eine Syntaxkategorie ist, desto unähnlicher sind sich diejenigen Syntagmen, die die Elemente dieser Kategorie bilden. Das scheint paradox, doch es stimmt mit der bekannten Tatsache überein, daß man ganze Sätze in Konstituenten einbetten kann, die ihrerseits in der Regel als Konstituenten von Sätzen auftreten.

Dasjenige, was eine syntaktische Kategorie zu einer solchen macht, kann also kein homogenes Prinzip der Mengenbildung sein. Vielmehr muß es auf Ähnlichkeiten beruhen, und Ähnlichkeit ist keine transitive Relation. Nimmt man es extensional, so heißt dies, daß syntaktische Kategorien nicht durchgängig disjunkt sein können. Es muß also grundsätzlich möglich sein, daß eine Konstituente nicht eindeutig einer syntaktischen Kategorie zugeordnet werden kann. Der empirischen Syntax-Forschung ist dies ein vertrautes Phänomen. Es spiegelt sich in den bekannten Schwankungen der Orthographie. Dann aber ist das von Goodman beschriebene Flottieren von Erfüllungsgegenständen zwischen Schemata im Bereich der Syntax grundsätzlich an jeder Stelle möglich, ${ }^{85)}$ und über Zwischenschritte vermittelt wird dann auch zwischen den extremsten syntaktischen Gegensätzen keine strikte Grenze bestehen. Nomina können zu Verben werden und umgekehrt. Die diachronische Sprachbeschreibung erhält in dieser Perspektive einen präzisen empirischen Sinn: Hierbei handelt es sich ja in der Regel um langfristige Prozesse. ${ }^{86}$

Was man allgemein als den Fluktuanz-Charakter der Sprache be-

84) Ich gehe hier - aus einsichtigen Gründen - von einer „Oberflächensyntax“ aus. Tiefenstrukturkonstrukte wie eine Auxiliarkategorie berücksichtige ich hier nicht.

85) Vgl. Goodman 1997, S. 146 f.86 Ein Modellfall wäre hier etwa die Herausbildung der Endstellung des Finitums im Deutschen. 
schreiben kann, muß also in der Beschreibung einzelner Sprachformen so eingelöst werden, daß dieser Charakter an der Beschreibung der Elemente jeder einzelnen Form sichtbar gemacht werden kann. Damit ist man aber letztlich auf die Ebene der kognitiven Prozesse verwiesen, die aus einer Menge „nur “ähnlicher Elemente eine Kategorie zu machen vermögen.

Von der Linguistik erfordert dies erstens eine völlig neuartige Empirie, die nur im Zusammenspiel mit der Psychologie zu erreichen ist. Denn die Homogenität einer Sprachkategorie kann dann nur noch in den Verarbeitungsprozessen liegen, die aus dem heterogenen Material ein Ganzes machen. Die Beschreibung des Wertes des signe linguistique ist, so paradox dies klingen mag, nicht mehr Sache der Linguistik. Diese wird ihn exemplifizieren können, an ausgewählten Beispielen erläutern, doch seine allgemeine Repräsentation ist Sache der Psychologie.

Die zweite Anforderung an die Linguistik, die erfüllt werden muß, um überhaupt mit der Nachbardisziplin ins interdisziplinäre Gespräch eintreten zu können, bestünde dann darin, sich über die implizite Logik der in der eigenen Disziplin gebräuchlichen Repräsentationsapparate Klarheit zu verschaffen. In dieser Hinsicht ist es der nach-chomskyschen Linguistik aufgegeben, eine formale Disziplin zu werden.

\section{Literatur:}

Borsche, Tilman(1981): Sprachansichten. Der Begriff der menschlichen Rede in der Sprachphilosophie Wilhelm von Humboldts. Stuttgart: Klett-Cotta.

Borsche, Tilman, Hg. (1996): Klassiker der Sprachphilosophie. Von Platon bis Noam Chomsky. München: Beck.

Di Cesare, Donatella (1996): Wilhelm von Humboldt. In: Tilman Borsche, Hg. (1996). S. $275-289$.

Eisenberg, Peter(1998): Grundriß der deutschen Grammatik. Band I: Das Wort. Stuttgart, Weimar: Metzler.

Goodman, Nelson(1997): Sprachen der Kunst. Entwurf einer Symboltheorie. Übersetzt von Bernd Philippi. Frankfurt a. M.: Suhrkamp (= stw 1304)

Goodman, Nelson, und Elgin, Catherine Z. (1989): Revisionen: Philosophie und

86) Ein Modellfall wäre hier etwa die Herausbildung der Endstellung des Finitums im Deutschen. 
andere Künste und Wissenschaften. Frankfurt a. M.: Suhrkamp (= stw 1050). Grewendorf, Günther(1995): Sprache als Organ - Sprache als Lebensform. Anhang: Interview mit Noam Chomsky: Über Linguistik und Politik. Frankfurt a. M.: Suhrkamp.

Herder, Johann G. [1985]: Frühe Schriften 1764 - 1772. Hg. von Ulrich Gaier. Frankfurt a.M.: Dt. Klassiker Vlg $(=$ Werke in zehn Bänden, hg. von Martin Bollacher u. a., Bd. 1). Zitiert als Frühe Schriften.

Humboldt, Wilhelm von [1968]: Gesammelte Schriften. 17 Bde. Hgg. von Albert Leitzmann u.a. Berlin 1903 ff. Photomech. Nachdr. Berlin 1968. Zitiert als GS.

Ders. [1960 ff.]: Werke in fünf Bänden. Hgg. von. Andreas Flitner und Klaus Giel. Darmstadt: Wiss. Buchges. Zitiert als WW.

Jakobson, Roman (1969): Kindersprache, Aphasie und allgemeine Lautgesetze. Frankfurt a. M.: Suhrkamp.

Leroi-Gourhan, André(1984): Hand und Wort. Die Evolution von Technik, Sprache und Kunst. 2. Aufl. Frankfurt a. M.: Suhrkamp.

Lorenz, Kuno und Mittelstraß, Jürgen (1967): On Rational Philosophy of Language: The Programme in Plato's Cratylus Reconsidered. In: Mind 76, No. 301, 1967, S. $1-20$.

Putnam, Hilary (1991): Repräsentation und Realität. Übersetzt von Joachim Schulte. Frankfurt a. M.: Suhrkamp.

de Saussure, Ferdinand (1967): Cours de linguistique générale. Édition critique par Rudolf Engler. Wiesbaden $1967 \mathrm{ff} .:$ Harrassowitz. Zitiert als EC.

Ders. (1972): Cours de linguistique générale. Édition critique préparée par Tullio de Mauro. Paris: Payot. Zitiert als CLG.

Schneider, Hans J.(1992): Phantasie und Kalkuil. Über die Polarität von Handlung und Struktur in der Sprache. Frankfurt a. M.: Suhrkamp.

Spitzer, Manfred (1996): Geist im Netz. Modelle für Lernen, Denken und Handeln. Darmstadt: Wiss. Buchges.

Stetter, Christian (1992): Ferdinand de Saussure. In: Marcelo Dascal, Dietfried Gerhardus, Kuno Lorenz und Georg Meggle, Hg. (1992): Sprachphilosophie. Ein internationales Handbuch zeitgenössischer Forschung. Berlin - New York: de Gruyter. 1. Halbbd. S. 510 - 523.

Ders. (1999): Der Käfer in der Schachtel: Das Privatsprachenproblem und die Universalgrammatik. In: Lili 29, 1999, Heft 115, S. 37 - 66.

Ders. (2001): Am Ende des Chomsky-Paradigmas - zurück zu Saussure? In: Cahiers Ferdinand de Saussure 54, 2001, S. 219 - 267.

Ders. (2002): Linguistische Ästhetik: Zum mimetischen und logischen Gebraquch 
der Schrift. In: Vittoria Borsò u. a., Hgg. (2002): Schriftgedächtnis Schriftkulturen. Stuttgart und Weimer: Metzler. S. 219-237.

Tugendhat, Ernst (1988): TI KATA TINO $\Sigma$. Eine Untersuchung zu Struktur und Ursprung aristotelischer Grundbegriffe. 4., unveränderte Aufl. Freiburg und München: Alber.(1. Aufl. 1958.) 